\title{
A Multi-source Information Fusion Approach in Tunnel Collapse Risk Analysis based on Improved Dempster-Shafer Evidence Theory
}

Bo Wu

Guangxi University

Weixing Qiu ( $\sim 1910302047 @ s t . g x u . e d u . c n)$

Guangxi University

Wei Huang

Guangxi University

Guowang Meng

Guangxi University

Jingsong Huang

Guangxi University

ShiXiang Xu

Guangxi University

\section{Research Article}

Keywords: Cloud model, Bayesian network, Support vector machine, D-S evidence theory, Tunnel collapse, Safety risk assessment

Posted Date: October 27th, 2021

DOI: https://doi.org/10.21203/rs.3.rs-1002621/v1

License: (c) (1) This work is licensed under a Creative Commons Attribution 4.0 International License.

Read Full License

Version of Record: A version of this preprint was published at Scientific Reports on March 7th, 2022. See the published version at https://doi.org/10.1038/s41598-022-07171-x. 


\section{A multi-source information fusion approach in tunnel collapse}

risk analysis based on improved Dempster-Shafer evidence

\section{theory}

Bo Wu ${ }^{1,2,3}$, Weixing $\mathrm{Qiu}^{1,{ }^{*}}$, Wei Huang ${ }^{1}$, Guowang Meng ${ }^{1}$, Jingsong Huang ${ }^{1}$, ShiXiang $\mathrm{Xu}^{1}$

1. College of Civil Engineering and Architecture, Guangxi University, 100 University Road, Nanning, Guangxi 530004, China

2. School of Civil and Architectural Engineering, East China University of Technology, Nanchang 330013, Jiangxi, China

3. School of Architectural Engineering, Guangzhou City Construction College, Guangzhou 510925, Guangdong, China

*Corresponding author.

E-mail addresses: wubo@gxu.edu.cn(B.Wu),1910302047@st.gxu.edu.cn (W. Qiu), wei.huang@st.gxu.edu.cn (W. Huang),menggwang@163.com(G. Meng),164502262@,qq.com (J. Huang), 603559081@qq.com (S.Xu)

Abstract: The tunneling collapse is the main engineering hazard in the construction of the drillingand-blasting method. The accurate assessment of the tunneling collapse risk has become a key issue in tunnel construction. As for assessing the tunneling collapse risk and providing basic risk controlling strategies, this research proposes a novel multi-source information fusion approach that combines Bayesian network (BN), cloud model (CM), support vector machine (SVM), DempsterShafer (D-S) evidence theory, and Monte Carlo (MC) simulation technique. Those methods (CM, BN, SVM) are used to analyze multi-source information (i.e. statistical data, physical sensors, and expert judgment provided by humans) respectively and construct basic probability assignments (BPAs) of input factors under different risk states. Then, these BPAs will be merged at the decision level to achieve an overall risk evaluation, using an improved D-S evidence theory. The MC technology is proposed to simulate the uncertainty and randomness of data. The novel approach has 
been successfully applied in the case of the Jinzhupa tunnel of the Pu-Yan Highway (Fujian, China). The results indicate that the developed new multi-source information fusion method is feasible for (a) Fusing multi-source information effectively from different models with a high-risk assessment accuracy of $98.1 \%$; (b) Performing strong robustness to bias, which can achieve acceptable risk assessment accuracy even under a 20\% bias; and (c) Exhibiting a more outstanding risk assessment performance ( $97.9 \%$ accuracy) than the single-information model $(78.8 \%$ accuracy) under a high bias $(20 \%)$. Since the proposed reliable risk analysis method can efficiently integrate multi-source information with conflicts, uncertainties, and bias, it provides an in-depth analysis of the tunnel collapse and the most critical risk factors, and then appropriate remedial measures can be taken at an early stage.

Keywords: Cloud model; Bayesian network; Support vector machine; D-S evidence theory; Tunnel collapse; Safety risk assessment

\section{Introduction}

The highways are extremely important infrastructures for most countries. It ensures communication and development between different regions, especially in the mountains and hilly areas. Most of the surrounding rocks of highway tunnels are mainly hard rock mass, and the geological conditions of the crossing sections are complex and changeable (Sun, 2019). Hard rock tunnels are mostly constructed by drilling and blasting. Due to various risk factors in the complex project environment, safety violations often occur in highway tunnel construction. The collapse is one of the most frequent and harmful geological hazards during the construction of a tunnel. Because the collapse was sudden and instantaneous, it was difficult to predict and the construction workers 
did not have enough time to escape. Once the tunnel collapse occurs, it may cause serious economic losses, construction delays, and even human casualties. Therefore, it is necessary to research the risk mechanism of tunnel collapse by considering the accident scenario and safety analysis, aiming to provide decision support for assuring the safety of tunnel construction.

In recent years, a lot of research work has been carried out in tunnel collapse risk assessment. Zhou (Zhou, 2008) proposed a method for tunnel collapse risk analysis based on the fuzzy Analytic Hierarchy Process. He discussed the collapse mechanism of mountain tunnels and proposed a list of risk factors for tunnel collapse. The Bayesian network is used to conduct a quantitative analysis of safety risks in the Wuhan Yangtze River Metro Tunnel (Zhang, 2014). Wu et al. (Wu et al., 2015) proposed an evaluation method based on a dynamic Bayesian network to provide a real-time dynamic risk assessment for tunnel construction. An optimization method for the preliminary support parameters was proposed based on the genetic algorithm (GA) and combined covariance Gaussian process regression (CCGPR) coupled algorithm presented to provide a complete information-based construction method for tunnel engineering (Liu and Liu, 2019). There are also many studies using artificial intelligence for risk assessment to realize the automation and intelligence of assessment. Pan (Pan and Zhang, 2021) used artificial intelligence to monitor the entire life cycle of real complex projects. The artificial neural networks are used to assess the risk of shield drilling under severe ground conditions such as squeezing grounds (Hasanpour et al., 2020). However, since the above evaluation methods only focus on a single information source, the reliability and accuracy of the security risk assessment cannot be guaranteed. Incomplete consideration of information can lead to inaccurate assessment results, which can not provide accurate recommendations to decision makers (Guo and Zhang, 2021). This would defeat the 
purpose of the risk assessment. In comparison, the fusion model can greatly improve the accuracy of prediction results due to it has a better understanding of risk factors (Pan et al., 2020). For example, a fusion model is proposed to predict the risk of water inrush disasters (Li et al., 2021). The fusion of sensor data and simulation data improves the accuracy of the structural safety risk assessment (Pan et al., 2020). Nowadays, there has been an increasing interest in the development of modern information technology and Internet technology, which makes the processing and analysis of data from multiple sources particularly important. The data fusion technology may prove to be more helpful to meet the security risk management needs of the tunnel construction than point-based methods (Ding and Zhou, 2013). This research proposes a novel risk assessment approach that integrates Monte-Carlo (MC) simulation technique, normal cloud model (CM), Bayesian networks (BN), probabilistic support vector machine (SVM), and D-S evidence theory. The tunneling collapse risk probability distribution is obtained by analyzing different information sources with different models. Finally, the judgment of each model is fused to give the overall collapse risk result. This model aims to achieve the following goals: (1) Constructing models to estimate the collapse risk according to the expert judgment, monitoring data, and tunneling collapse database; (2) The judgment of the models is fused to get the final collapse risk assessment result; (3) Evaluating the performance of the models to quantify the quality of judgment.

\section{Literature review}

\subsection{Dempster-Shafer (D-S) evidence theory}

Information sources are usually divided into three categories, namely statistical data, physical 
sensors, and expert judgment provided by humans(Yager, 2016). Among them, statistical data and physical sensors are called hard information. Humans act as soft sensors and execute decisionmaking processes through a web-based system (Balazs and Velásquez, 2016). Regarding evidence, each source of information constitutes all the evidence on which the decision is based (Leung et al., 2013). In the complex decision-making process, how to compose multiple sources of evidence that may conflict with each other has become a challenging task. So far, over the years, various information fusion researches have been proposed, such as rough set (Qian et al., 2010; Zhang et al., 2021), Dempster-Shafer (D-S) evidence theory (Guo and Zhang, 2021; Leung et al., 2013), maximum entropy approach (Yager, 2016), and others. Among the above-mentioned information fusion methods, D-S evidence theory is an effective and common method in the field of information fusion. Pan et al. (Pan et al., 2020) proposed a risk analysis method based on SVM and D-S evidence theory to fuse different monitoring data, in order to evaluate the structural health status. Zhang et al. (Zhang et al., 2017) developed a novel safety risk assessment method based on D-S evidence theory and the cloud model to perceive the safety risk of buildings adjacent to the tunneling excavation.

However, the traditional D-S evidence theory cannot deal with highly conflicting evidence and will lead to unexpected and counter-intuitive results and make the evidence fusion approach insignificant (Zhang et al., 2017). In order to minimize the negative effect of high-conflict evidence, this paper adopted an improved D-S evidence theory by combining the weighted mean rule and the D-S evidence theory to solve the above problem.

\subsection{Classification method}

For classification problems, support vector machines (SVM) and artificial neural networks 
112 (ANN) are the two main supervised learning algorithms in the field of machine learning(Worden

113 and Manson, 2007). Although ANN has provided a powerful tool for the research on tunnel

114 construction (Satpal et al., 2016; Zhou et al., 2016). There are still limitations such as long

115 calculation time, spatial disasters, local minima, overfitting, etc. (Huang, 2009; Pan and Zhang,

116 2021). Due to the small sample of training data for the tunneling collapse case in this paper,

117 classification using neural networks will be prone to overfitting. The support vector machines

118 (SVM), as a method parallel to artificial neural networks (ANN), is a machine learning method

119 established based on the principle of structural risk minimization and the statistical learning theory

120 for a small sample. The SVM has higher accuracy in a small number of training data predictions.

121 Therefore, this article attempts to use SVM to process statistical data for collapse risk assessment.

\section{3. Methodology}

In order to improve the credibility and robustness of the tunnel collapse risk evaluation, a new

hybrid multi-source information fusion method is proposed. Fig. 1 is a flowchart of the tunnel collapse risk analysis method in this paper. In the developed method, all available data from the construction process is collected for risk analysis to improve the accuracy and robustness of the refine and synthesize different classification results generated from probabilistic models. According to the characteristics of different information sources, choose the corresponding probability model. 
133 and probability theory to map qualitative concepts and quantitative data and is therefore used to

134 process monitoring data during the constructs of BPAs. For expert judgment provided by humans,

135 Bayesian Networks (BN) is used to investigate causal relationships between tunnel collapse and its

136 influential variables based upon the risk/hazard mechanism analysis and expert scores.

137 In collapse risk assessment, the MC simulation can conduct risk analysis by constructing a

138 calculation model containing a series of inherent uncertain variables (Robert and Casella, 2004). It

139 can estimate all possible decision results and evaluate the impact of risks in an uncertain

140 environment (Haroonabadi and Haghifam, 2011). The MC simulation is adopted to simulate

141 measurement and human error, proving the robustness of the hybrid approach. A typical hazard

142 concerning the tunnel collapse in the construction of the Fujian Jinzhupa Tunnel in China is

143 presented as a case study. The results demonstrate the feasibility of the proposed approach and its

144 application potential. 


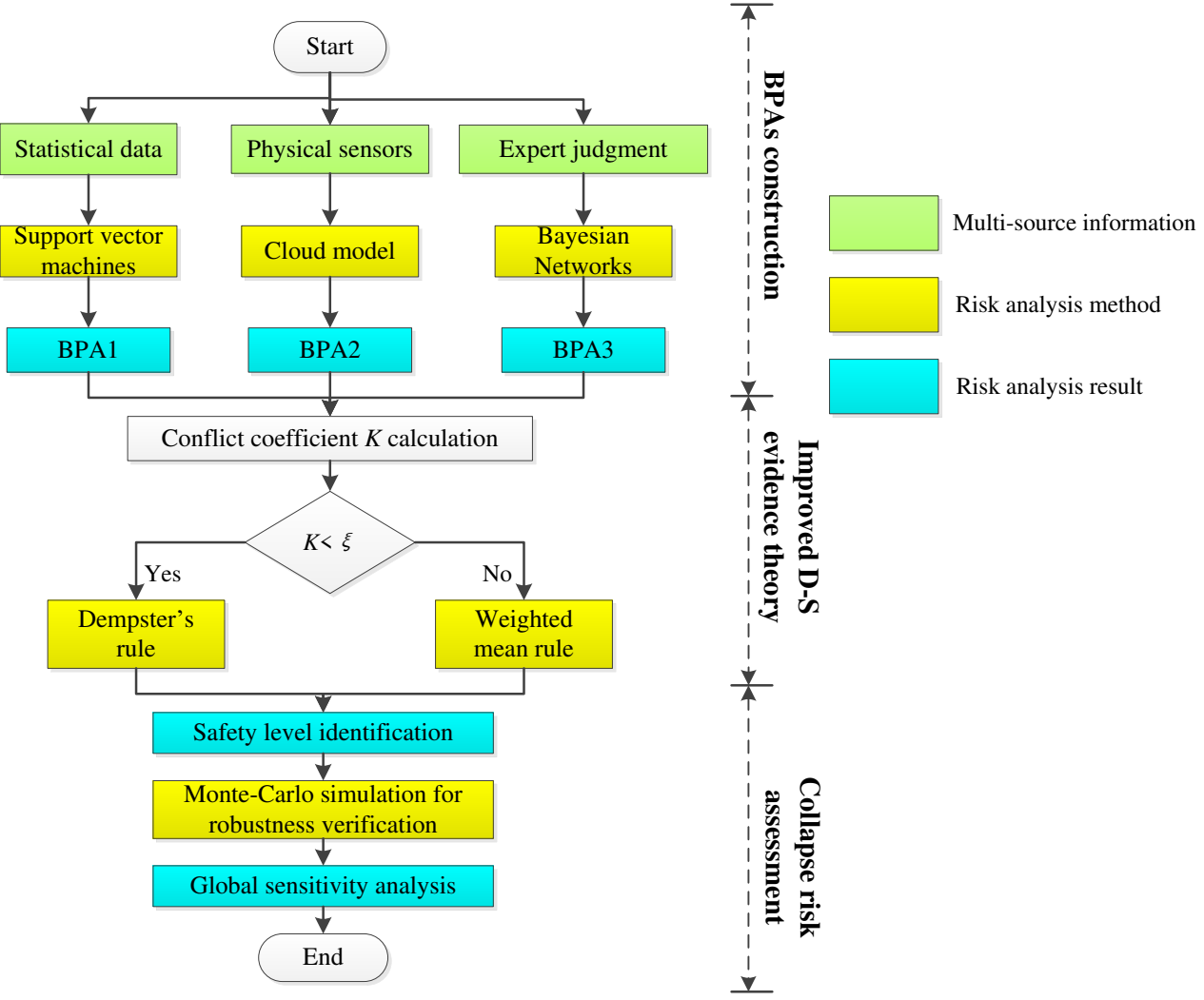

Fig. 1 Flowchart of the proposed hybrid method for multi-source information fusion decision.

\subsection{BPA construction}

\subsubsection{Normal cloud model}

The normal cloud model is a new cognition model of uncertainty, proposed by $\mathrm{Li}$ et al. ( $\mathrm{Li}$ et al., 2009). It can synthetically describe the randomness and fuzziness of elements and implement the uncertain transformation between a qualitative concept and its quantitative value. The normal $\mathrm{CM}$ can be determined by numerical characteristics $(E x, E n, H e)$. The Expectation " $E x$ " is the expectation of the cloud droplets in the universe of discourse and the typical sample of a qualitative concept. The Entropy " $E n$ " is the entropy of " $E x$ ", representing the uncertainty measurement of a qualitative concept. Hyper-entropy "He" represents the uncertainty degree of Entropy " $E n$ ".

Let $X$ be the universe of discourse and $B$ be a qualitative concept connected with $X$. If there is 
157

a number $x,(1) x \in X,(2) x$ is a random instantiation of concept $B,(3) x$ satisfies Eq. (1), the grade of a certain degree of $x$ belonging to concept $B$ satisfies Eq. (2) (Li et al., 2009):

$$
\begin{gathered}
\left\{\begin{array}{c}
x: N\left(E x, E n^{\prime 2}\right) \\
E n^{\prime}: N\left(E n, H e^{2}\right)
\end{array}\right. \\
\mu(x)=e^{-\frac{(x-E x)^{2}}{2\left(E n^{\prime}\right)^{2}}}
\end{gathered}
$$

The tunneling collapse risk assessment is a multi-source information decision-making problem under uncertain conditions. Various tunnel collapse risk factors $B_{i}$ are analyzed in the decisionmaking process. In order to explore useful information from multiple sources, each risk factor should be further divided into different risk states $B_{i j}(i=1,2, \ldots, M ; j=1,2, \ldots, N)$. Each risk state can correspond to a specific double limit interval, denoted as $\left[b_{i j}(L), b_{i j}(R)\right]$. The conversion from the double limit interval $\left[b_{i j}(L), b_{i j}(R)\right]$ to the normal cloud model $\left(E x_{i j}, E n_{i j}, H e_{i j}\right)$ can be achieved by Eq. (3).

$$
\left\{\begin{aligned}
E x_{i j}= & \frac{b_{i j}(L)+b_{i j}(R)}{2} \\
E n_{i j}= & \frac{b_{i j}(R)-b_{i j}(L)}{6}, \quad(i=1,2, \ldots, M ; j=1,2, \ldots, N) \\
& H e_{i j}=h
\end{aligned}\right.
$$

where, " $E x_{i j}$ " is the expectation; " $E n_{i j}$ " is the entropy of " $E x_{i j}$ ", " $H e_{i j}$ " is the Hyper-entropy. The range of the constant " $h$ " is from 0 to " $E n_{i j}$ " which is adapted to reflects the uncertainty degree of those factors.

In the $\mathrm{CM}$ framework, the correlation can measure the relative membership between the observed value $b_{i j}$ of the factor $B_{i}$ and the cloud model of a specific risk state $B_{i j}$. The measurement of BPAs under different risk states of influential factors can be obtained by Eq. (4) (Zhang et al., 2017). 


$$
\left\{\begin{array}{c}
m_{i}\left(B_{j}\right)=\exp \left(-\frac{\left(x_{i}-E x_{i j}\right)^{2}}{2\left(E n_{i j}^{\prime}\right)^{2}}\right),(i=1,2, \ldots, M ; j=1,2, \ldots, N) \\
m_{i}(\Phi)=1-\sum_{j=1}^{N} m_{i}\left(A_{j}\right)
\end{array}\right.
$$

where, $m_{i}\left(B_{j}\right)$ is the belief measure; $E n^{\prime}$ represents a random number that satisfies $E n^{\prime}: N\left(E n, H e^{2}\right)$, and $m_{i}(\Phi)$ represents the BPAs value in uncertain situations, that is, the focus element cannot be determined under the indicator $B_{i}$, so all elements are included.

\subsubsection{Probabilistic SVM}

The traditional linear SVM performs linear division by a hyperplane. This hyperplane is found by maximizing the separation margin, which is the distance between the hyperplane and the closest data point. The kernel function is used to map the original data from a low-dimensional space to a feature space with a high-dimensional space, which can obtain better classification accuracy. Besides, the penalty parameter $C$ of the error term also plays a key role in classification accuracy. A high value of $C$ means a strict classifier that does not admit many misclassified points. (Liu et al., 2014). The discrimination function is:

$$
f(x)=\operatorname{sign}\left[\left(\sum_{i=1}^{m} \alpha_{i} y_{i} K\left(x_{i}, x\right)\right)+b\right]
$$

where $m$ is the size of the training data set, $\alpha_{i}$ represents Lagrange multipliers, $K\left(x_{i}, x\right)$ is a kernel function, and $b$ is a threshold parameter based on the training set.

The linear SVM only gives one class prediction output that will be either yes or no. To extract the associated probabilities from SVM outputs, several methods have been proposed. This research chooses Platt's approach (J. Platt, 1999), which uses the Sigmoid function to map the output of the 
SVM to the interval $[0,1]$, as given by Eq. (6).

$$
P(y=1 \mid x) \approx P_{a b}(f(x))=\frac{1}{1+e^{(a f(x)+b)}}
$$

where $a$ and $b$ are the parameters computed from the minimization of the negative log-likelihood function on a set of training examples:

$$
\begin{aligned}
& \min _{z=(A, B)} F(z)=-\sum_{i=1}^{l}\left(t_{i} \log \left(p_{i}\right)+\left(1-t_{i}\right) \log \left(1-p_{i}\right)\right), \\
& \left\{\begin{array}{l}
t_{+}=\frac{N_{+}+1}{N_{+}+2} \\
t_{-}=\frac{1}{N_{-}+2}
\end{array} i=1,2, \mathrm{~K}, l\right.
\end{aligned}
$$

where $t_{i}$ is the new label of the classes: +1 becomes $t_{+}$and -1 becomes $t_{-}, N_{+}$and $N_{-}$are the number of points that belong to class 1 and class 2 respectively.

\subsubsection{Bayesian network}

The Bayesian network $(\mathrm{BN})$ is a combination of two different mathematical areas, the probability theory, and graph theory. It consists of several conditional probability tables (CPT) and a directed acyclic graph (DAG) (Li et al., 2017). A BN model with $\mathrm{n}$ nodes can be represented as $B\langle G, \Theta\rangle$, where $\mathrm{G}$ stands for a DAG with n nodes and $\Theta$ is defined as the CPT of the BN model. A general BN intuitively represents a complex network with $n$ nodes and direct edges. The nodes $\left\{X_{1}, \mathrm{~L}, X_{n}\right\}$ in the graph are labeled by related random variables. The directed edges between nodes represent the relationship between variables. Each node is attached to a CPT that contains the conditional probability of the parent node.

Assuming parents $\left(X_{i}\right)$ is the parent nodes of $X_{i}$ in DAG, the conditional probability distribution of $X_{i}$ is defined as $P\left(X_{i} \mid\right.$ parents $\left.\left(X_{i}\right)\right)$. The calculation of $P(x)$ can be written as Eq. (8). 


$$
P(x)=P\left(X_{1}, \mathrm{~L}, X_{n}\right)=\prod_{X_{i} \in\left\{X_{1}, \mathrm{~L}, X_{n}\right\}} P\left(X_{i} \mid \operatorname{parents}\left(X_{i}\right)\right)
$$
al., 2017).

\subsection{Improved D-S evidence theory}

In this paper, the D-S theory is used to combine multi-source information to obtain the tunnel collapse risk. Dempster's combinational rule for multiple evidence is calculated with Eq. (9).

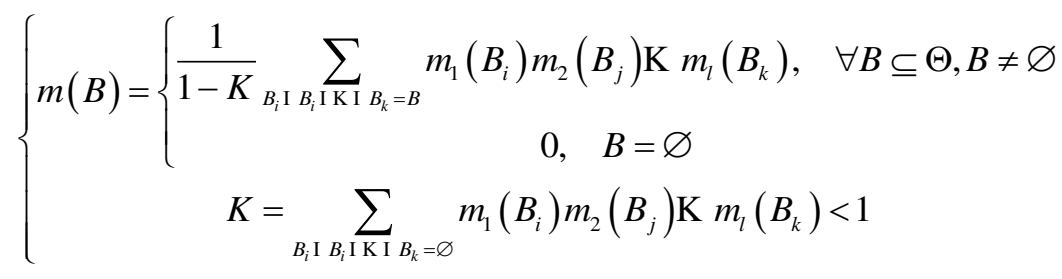

where $K$ is defined to be the normalization factor. $l$ is the number of evidence pieces in the process of combination, and $i, j, k$ denotes the $i$ th, $j$ th, and $k$ th hypothesis, respectively.

When the value of $K$ is close to 1 , there will be a high conflict, which means that Dempster's evidence aggregation rule will be meaningless. To deal with high-conflict evidence, this paper proposed a hybrid combination rule by combining the weighted mean rule and the Dempster's rule. This article will use a threshold $\xi$ to indicate high evidence conflicts. When $K$ is greater than $\xi$, there is high evidence conflict, and the D-S evidence theory will be replaced by the weighted mean rule, as shown in Eq. (10). In this research, the value of the threshold $\xi$ is defined to be 0.95 (Zhang et

$$
\begin{gathered}
d=\sum_{j=1}^{j=l} \sqrt{\sum_{k=1}^{k=L}\left(m_{i}\left(B_{k}\right)-m_{j}\left(B_{k}\right)\right)^{2}} \\
w_{i}=\frac{d_{i}^{-1}}{\sum_{i=1}^{i=l} d_{i}^{-1}} \\
\left\{\begin{array}{l}
m_{i}^{*}\left(B_{k}\right)=w_{i} \mathrm{~g} m_{i}\left(B_{k}\right) \\
m_{i}(\Theta)=1-\sum_{k=1}^{L} m_{i}^{*}\left(B_{k}\right)
\end{array}\right.
\end{gathered}
$$


where $l$ and $L$ are the numbers of evidence and the number of hypotheses, respectively, and $k$ is the $k$ th hypothesis.

\subsection{Tunnel collapse risk assessment}

The collapse risk assessment can provide support for construction decision-making on site.

Once the collapse risk drops to a high-risk level, certain precautions can be taken before the tunnel collapses. After multiple information sources are fused at the decision-making level, the result of tunnel collapse risk assessment depends on the maximum value of BPAs, as shown in Eq. (11). The confidence indicator $m_{i}(\Theta)$ is designed to measure the credibility of the fusion result.

$$
\left\{\begin{array}{c}
m\left(B_{w}\right)=\max \left\{m\left(B_{i}\right)\right\} \\
m(\Theta)<\theta \quad \theta=0.1
\end{array}\right.
$$

where $B_{i}$ denotes collapse risk levels, $B_{w}$ indicates the probability of different risk levels $m(B)=\left\{m\left(B_{1}\right), m\left(B_{2}\right), \ldots, m\left(B_{n}\right), m(\Theta)\right\}$

The sensitivity analysis of the tunneling collapse risk factors is proposed to reveal the sensitivity of system performance to small changes in risk factors. Up to now, some sensitivity analysis methods have been proposed (Janssen, 2013). To consider the nonlinearity and interaction relationship between risk factors, this paper adopts global sensitivity analysis (GSA). Spearman's rank correlation coefficient (a GSA measure) does not depend on distributions with a similar shape or being linearly related (Kou et al., 2012). The GSA measurement of the $i$ th input factor $C_{i}$ can be calculated by Eq. (12).

$$
G S A\left(C_{i}\right)=\frac{\sum_{p=1}^{P}\left(R\left(x_{i}^{p}\right)-\bar{R}\left(x_{i}^{p}\right)\right)\left(R\left(t^{p}\right)-\bar{R}\left(t^{p}\right)\right)}{\sqrt{\sum_{p=1}^{P}\left(R\left(x_{i}^{p}\right)-\bar{R}\left(x_{i}^{p}\right)\right)^{2}} \sqrt{\sum_{p=1}^{P}\left(R\left(t^{p}\right)-\bar{R}\left(t^{p}\right)\right)^{2}}}
$$

247 where $P$ is the number of the repeated interactions; $R\left(x_{i}^{p}\right)\left(R\left(t^{p}\right)\right)$ is the rank of $x_{i}^{p}\left(t^{p}\right)$ among 
the simulated input data; $\bar{R}\left(x_{i}^{p}\right)\left(\bar{R}\left(t^{p}\right)\right)$ is the mean value of $R\left(x_{i}^{p}\right)\left(R\left(t^{p}\right)\right)$.

\section{A case study}

The Jinzhupa Tunnel is a twin-tube highway tunnel. The right and left tunnels are $782 \mathrm{~m}$ and $771 \mathrm{~m}$ long, respectively. This paper takes the left line $(\mathrm{ZK} 242+548 \sim Z K 243+319)$ as the object of study. The fault structure along the left line of the tunnel is shown in Fig. 2. There are $316 \mathrm{~m}$ of Vlevel surrounding rock section and $455 \mathrm{~m}$ of IV-level surrounding rock section. The rock mass is mainly composed of the residual silty clay, granite fully weathered layer, and broken strong weathered layer. Furthermore, there is a fracture fragmentation zone at section ZK243+139 160. Affected by this, the rock mass is relatively broken, showing a huge mosaic structure or broken mosaic structure. The rock mass is broken and has varying degrees of weathering. During the construction process, it is easy to cause tunnel collapse and water burst. Therefore, it is urgent to conduct a collapse risk assessment of the tunnel to reduce the losses caused by the collapse. In the proposed fusion method, the following four steps are adopted: set are used to train SVM models. information source, the cloud model is applied for collapse risk assessment. 

collapse risk value. further validated in the presence of unavoidable data biases. tunnel collapse risk factor is divided into four levels, as shown in Table 1.

Fig. 2 Fault structures along the Jinzhupa Tunnel

\section{Result and analysis}

\subsection{Collapse risk assessment based statistical data}

(1) Risk/hazard identification in the tunnel collapse

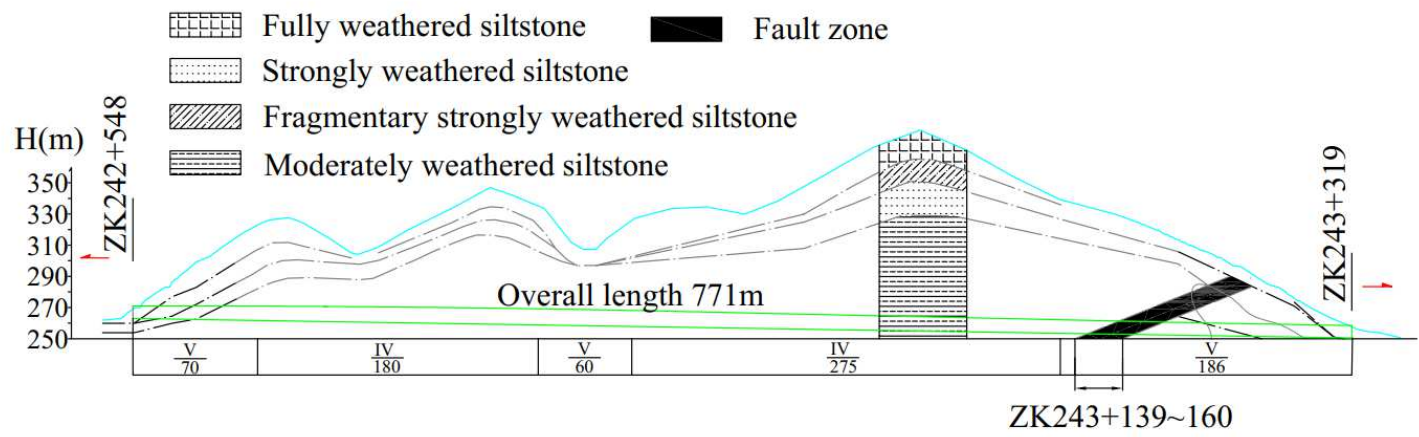

In actual engineering, the tunneling collapse may be affected by many factors, which interact with each other. Many scholars(Qiao et al., 2020; Wang et al., 2016, 2020; Zhang et al., 2020) have studied the risk factors of collapse and established a similar index system. Referring to previous researches, a total of 15 risk factors are selected, as shown in Table 1. The risk factors are analyzed in detail as shown in (Zhou, 2008; Zhang et al., 2020). At the same time, the safety status of each 
Table 1 Classified states of tunnel collapse risk factors.

\begin{tabular}{|c|c|c|c|c|}
\hline Factors & I & II & III & IV \\
\hline Tunnel collapse $(T)$ & Safe & Deformation & $\begin{array}{l}\text { Small-scale } \\
\text { collapse }\end{array}$ & $\begin{array}{l}\text { Large-scale } \\
\text { collapse }\end{array}$ \\
\hline Geometric factor $\left(B_{1}\right)$ & No risk & Low risk & Medium risk & High risk \\
\hline Geological factors $\left(B_{2}\right)$ & No risk & Low risk & Medium risk & High risk \\
\hline Construction technology $\left(B_{3}\right)$ & No risk & Low risk & Medium risk & High risk \\
\hline $\begin{array}{l}\text { Construction management } \\
\text { factors }\left(B_{4}\right)\end{array}$ & No risk & Low risk & Medium risk & High risk \\
\hline Excavation span $(\mathrm{m})\left(X_{1}\right)$ & $<7$ & $7-10$ & $10-14$ & $>15$ \\
\hline $\begin{array}{l}\text { Depth-to-height ratio }\left(H_{0} / H\right) \\
\qquad\left(X_{2}\right)\end{array}$ & $>20$ & $15 \sim 20$ & $10 \sim 15$ & $<10$ \\
\hline Rock mass grade $\left(X_{3}\right)$ & $\mathrm{I}(81 \sim 100)$ & $\mathrm{II}(61 \sim 80)$ & $\mathrm{III}(41 \sim 60)$ & IV, V $(<40)$ \\
\hline $\begin{array}{l}\text { Groundwater level } \\
\left(\left(H_{0}+H\right) / H_{\mathrm{w}}\right)\left(X_{4}\right)\end{array}$ & $<5$ & $5 \sim 20$ & $20 \sim 35$ & $>35$ \\
\hline Unfavorable geology $\left(X_{5}\right)$ & $\begin{array}{c}\text { Non- } \\
\text { Catastrophabilit } \\
\text { у }(76 \sim 100)\end{array}$ & $\begin{array}{c}\text { Weak } \\
\text { Catastrophabilit } \\
\text { у }(51 \sim 75)\end{array}$ & $\begin{array}{c}\text { Medium } \\
\text { Catastrophability } \\
(26 \sim 50)\end{array}$ & $\begin{array}{c}\text { Strong } \\
\text { Catastrophability } \\
(0 \sim 25)\end{array}$ \\
\hline Bias angle $\left({ }^{\circ}\right)\left(X_{6}\right)$ & $<10$ & $10 \sim 25$ & $25 \sim 40$ & $>40$ \\
\hline Primary support stiffness $\left(X_{7}\right)$ & Reasonable & $\begin{array}{c}\text { Almost } \\
\text { reasonable }\end{array}$ & Unreasonable & $\begin{array}{c}\text { Extremely } \\
\text { unreasonable }\end{array}$ \\
\hline $\begin{array}{c}\text { Ground reinforcement } \\
\text { measures }\left(X_{8}\right)\end{array}$ & Accurate & Almost accurate & Inaccurate & $\begin{array}{l}\text { Extremely } \\
\text { inaccurate }\end{array}$ \\
\hline Excavation method $\left(X_{9}\right)$ & CRD & $\mathrm{CD}$ & Bench & Full face \\
\hline $\begin{array}{l}\text { Waterproofing and drainage } \\
\text { measures }\left(X_{10}\right)\end{array}$ & Reasonable & $\begin{array}{c}\text { Almost } \\
\text { reasonable }\end{array}$ & Unreasonable & $\begin{array}{c}\text { Extremely } \\
\text { unreasonable }\end{array}$ \\
\hline $\begin{array}{l}\text { Timeliness of primary } \\
\text { support(min) }\left(X_{11}\right)\end{array}$ & $<30$ & $30 \sim 60$ & $60 \sim 120$ & $>120$ \\
\hline Monitoring $\left(X_{12}\right)$ & Reasonable & $\begin{array}{c}\text { Almost } \\
\text { reasonable }\end{array}$ & Unreasonable & $\begin{array}{c}\text { Extremely } \\
\text { unreasonable }\end{array}$ \\
\hline Construction quality $\left(X_{13}\right)$ & Good (76 100) & Fair $(51 \sim 75)$ & Poor $(26 \sim 50)$ & Very poor $(0 \sim 25)$ \\
\hline $\begin{array}{l}\text { Accuracy of geological } \\
\text { investigation }(\%)\left(X_{14}\right)\end{array}$ & $>90$ & $75 \sim 90$ & $60 \sim 75$ & $<60$ \\
\hline $\begin{array}{l}\text { Rationality of procedure } \\
\text { linkage }\left(X_{15}\right)\end{array}$ & Reasonable & $\begin{array}{c}\text { Almost } \\
\text { reasonable }\end{array}$ & Unreasonable & $\begin{array}{c}\text { Extremely } \\
\text { unreasonable }\end{array}$ \\
\hline
\end{tabular}

(2) Choice of kernel function and parameters

In order to construct the SVM model, a dataset of 70 tunnel collapses was collected from the

290 study (Zhou, 2008) and classified according to Table 1. The dataset is used as training data, and the

291 optimal hyperparameters $(C, \gamma)$ of the SVM model are found using the grid search method. Due to 


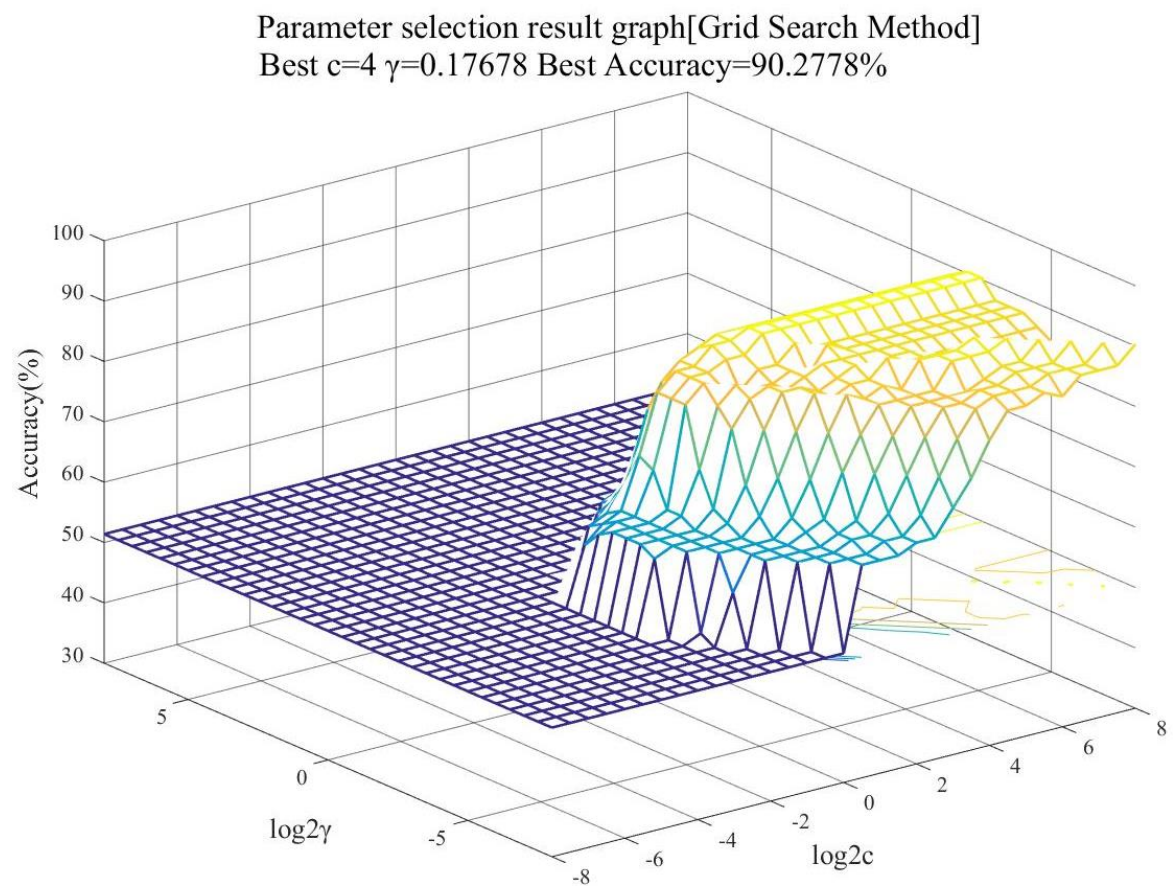

Fig. 3 Support Vector Machines evaluation accuracy based on pairs of $(C, \gamma)$.

(3) Calculation of the collapse risk probability

According to Eq. (6), the probability of different collapse risk levels is calculated. Since the

fracture zone is prone to collapse during excavation, this paper assesses the collapse risk of the risk value of the testing sample, the classification results as shown in Table 2. The risk level of tunnel collapse with the highest probability in the bold font in Table 2 represents the classification 
the second-highest probability is very close to the highest value in some of the prediction results.

$(0.50)$ is very close, which means that the results are very uncertain.

Table 2 Results of probabilistic Support Vector Machines.

\begin{tabular}{ccccccc}
\hline Tunnel section & $m(\mathrm{I})$ & $m(\mathrm{II})$ & $m(\mathrm{III})$ & $m(\mathrm{IV})$ & Predicted risk & Ture risk \\
\hline No.1 & 0.14 & $\mathbf{0 . 6 0}$ & 0.18 & 0.08 & II & II \\
No.2 & 0.02 & 0.02 & $\mathbf{0 . 9 4}$ & 0.02 & III & III \\
No.3 & 0.04 & $\mathbf{0 . 9 1}$ & 0.03 & 0.01 & II & II \\
No.4 & $\mathbf{0 . 6 4}$ & 0.30 & 0.06 & 0.00 & I & II \\
No.5 & 0.04 & $\mathbf{0 . 9 1}$ & 0.03 & 0.02 & II & II \\
No.6 & 0.03 & $\mathbf{0 . 9 1}$ & 0.04 & 0.02 & II & II \\
No.7 & 0.07 & $\mathbf{0 . 8 7}$ & 0.04 & 0.02 & II & II \\
No.8 & $\mathbf{0 . 9 1}$ & 0.03 & 0.03 & 0.03 & I & I \\
No.9 & 0.45 & $\mathbf{0 . 5 0}$ & 0.02 & 0.03 & II & I \\
No.10 & $\mathbf{0 . 8 3}$ & 0.09 & 0.04 & 0.04 & I & I \\
No.11 & 0.03 & $\mathbf{0 . 8 9}$ & 0.05 & 0.03 & II & II \\
No.12 & 0.01 & $\mathbf{0 . 9 3}$ & 0.01 & 0.05 & II & III \\
No.13 & $\mathbf{0 . 8 7}$ & 0.08 & 0.03 & 0.02 & I & I \\
No.14 & $\mathbf{0 . 9 2}$ & 0.04 & 0.02 & 0.02 & I & I \\
No.15 & 0.05 & $\mathbf{0 . 9 1}$ & 0.02 & 0.01 & II & II \\
No.16 & $\mathbf{0 . 8 7}$ & 0.08 & 0.02 & 0.02 & I & I \\
No.17 & 0.05 & $\mathbf{0 . 9 0}$ & 0.03 & 0.02 & II & II \\
No.18 & $\mathbf{0 . 8 6}$ & 0.08 & 0.03 & 0.03 & I & I \\
No.19 & $\mathbf{0 . 9 1}$ & 0.03 & 0.03 & 0.03 & I & I \\
No.20 & 0.03 & $\mathbf{0 . 9 1}$ & 0.04 & 0.02 & II & II \\
\hline
\end{tabular}

\subsection{Collapse risk assessment based on expert judgment}

312 (1) Establishment of the DAG and CPT

313 The DAG is mainly constructed by directed edges and node variables that represent the

314 probability causality between node variables. In combination with the risk factors in Table 1, the

315 DAG can be established, as shown in Fig. 4. To reduce the uncertainty of expert judgment, an expert

316 survey based on confidence index is used to construct the conditional probability tables (CPT), the detail as seen in (Zhang et al., 2020). 

the probability SVM, the accuracy of the model is lower. The second-highest probability in some prediction results is also very close to the highest value. For example, a tunnel section No.1, the probability of tunnel collapse for class II (0.48) and class III (0.52) is very close, which means that the results are very uncertain.

Similarly, taking the above 20 sections as examples, Eq. (8) is used to obtain the probability of their collapse risk value, the result as shown in Table 3. Compared with the prediction results of Table 3 Results of Bayesian network at ten monitoring sections.

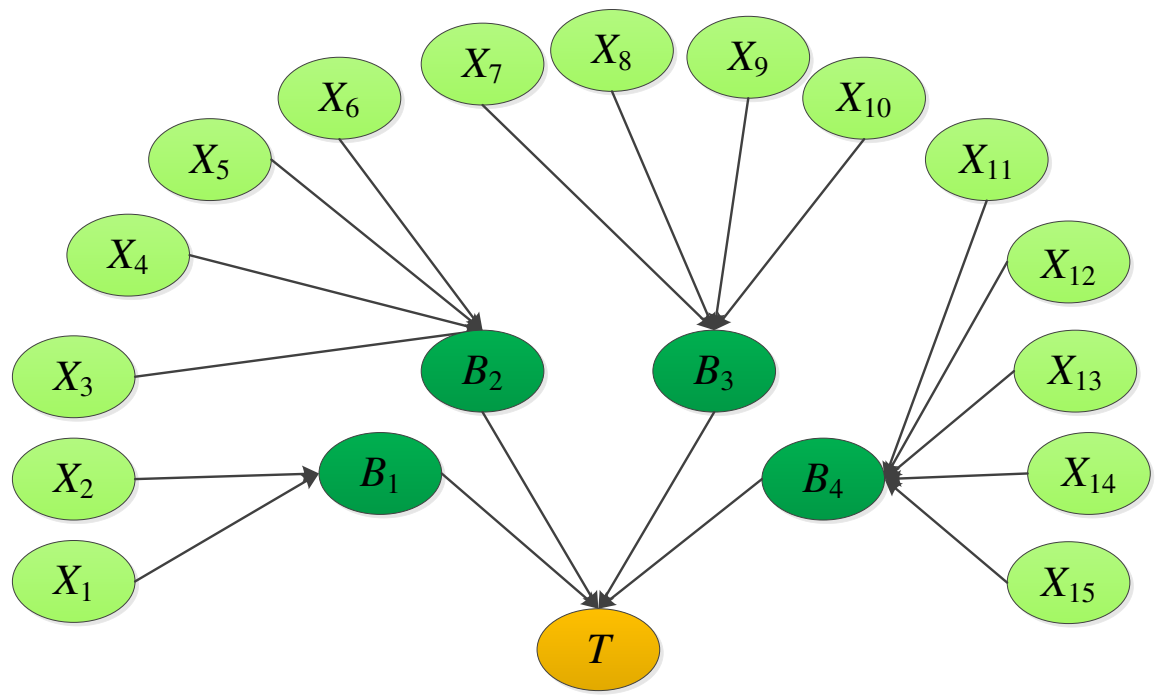

Fig. 4 DAG of Bayesian network.

\begin{tabular}{ccccccc}
\hline Tunnel section & $m(\mathrm{I})$ & $m(\mathrm{II})$ & $m(\mathrm{III})$ & $m(\mathrm{IV})$ & Predicted level & Ture level \\
\hline No.1 & 0.00 & 0.48 & $\mathbf{0 . 5 2}$ & 0.00 & III & II \\
No.2 & 0.00 & 0.00 & $\mathbf{0 . 9 3}$ & 0.07 & III & III \\
No.3 & 0.00 & $\mathbf{0 . 7 0}$ & 0.30 & 0.00 & II & II \\
No.4 & 0.00 & $\mathbf{0 . 9 6}$ & 0.04 & 0.00 & II & II \\
No.5 & 0.00 & $\mathbf{0 . 9 6}$ & 0.04 & 0.00 & II & II \\
No.6 & $\mathbf{0 . 7 8}$ & 0.22 & 0.00 & 0.00 & I & II \\
No.7 & 0.00 & $\mathbf{0 . 9 7}$ & 0.03 & 0.00 & II & II \\
No.8 & $\mathbf{0 . 8 4}$ & 0.16 & 0.00 & 0.00 & I & I \\
No.9 & 0.19 & $\mathbf{0 . 8 1}$ & 0.00 & 0.00 & II & I \\
No.10 & $\mathbf{0 . 8 7}$ & 0.13 & 0.00 & 0.00 & I & I \\
No.11 & 0.00 & $\mathbf{0 . 9 9}$ & 0.01 & 0.00 & II & II \\
No.12 & 0.00 & 0.01 & $\mathbf{0 . 8 3}$ & 0.17 & III & III \\
No.13 & $\mathbf{0 . 9 6}$ & 0.03 & 0.01 & 0.00 & I & I \\
No.14 & $\mathbf{0 . 6 5}$ & 0.35 & 0.00 & 0.00 & I & I \\
\hline
\end{tabular}




\begin{tabular}{lllllll}
\hline No.15 & 0.07 & $\mathbf{0 . 9 3}$ & 0.00 & 0.00 & II & II \\
No.16 & 0.33 & $\mathbf{0 . 6 7}$ & 0.00 & 0.00 & II & I \\
No.17 & 0.01 & $\mathbf{0 . 9 8}$ & 0.00 & 0.00 & II & II \\
No.18 & $\mathbf{0 . 6 6}$ & 0.33 & 0.01 & 0.00 & I & I \\
No.19 & 0.18 & $\mathbf{0 . 8 2}$ & 0.00 & 0.00 & II & I \\
No.20 & 0.00 & $\mathbf{0 . 9 3}$ & 0.07 & 0.00 & II & II \\
\hline
\end{tabular}

\subsection{Collapse risk assessment based on monitoring data indicator}

The monitoring measurement data includes the displacement of the vault, the surface settlement of the shallow buried section, and the change of the surrounding rock convergence. These data can reflect the stability of the tunnel support after the initial lining, thereby assessing the risk of collapse. Combined with this project, the vault displacement and the convergence displacement are used to analyze the collapse risk. According to the Chinese standards "Technical code for monitoring measurement of highway tunnel (DB 35/T 1067-2010)" and "Technical specification for construction of highway tunnel (JTG/T 3660-2020)", the daily deformation rate and cumulative deformation of the two-monitoring data are divided into four levels, as shown in Table 4.

Table 4 Classified states of monitoring measurement data.

\begin{tabular}{ccccc}
\hline Tunnel collapse level & I (Safe) & II (Deformation) & $\begin{array}{c}\text { III (Small-scale } \\
\text { collapse) }\end{array}$ & $\begin{array}{c}\text { IV (Large-scale } \\
\text { collapse) }\end{array}$ \\
\hline $\begin{array}{c}\text { Daily deformation } \\
\text { rate (mm/d) }\end{array}$ & $0 \leq x<2$ & $2 \leq x<5$ & $5 \leq x<10$ & $10 \leq x \leq 20$ \\
$\begin{array}{c}\text { Cumulative } \\
\text { deformation (mm) }\end{array}$ & $0 \leq y<50$ & $50 \leq y<100$ & $100 \leq y<200$ & $200 \leq y \leq 300$ \\
\hline
\end{tabular}

where, the cumulative deformation $(y)$ should be multiplied by the coefficient $(\zeta)$ according to the

340 distance between the measuring point and the excavation surface $(D)$, the detail as shown in Table

5. 


\begin{tabular}{ccccc}
\hline $\begin{array}{c}\text { The distance between the measuring point } \\
\text { and the excavation surface }(D)\end{array}$ & $1 B$ & $2 B$ & $3 B$ & $4 B \sim 4 B$ \\
\hline$\zeta$ & 0.5 & 0.75 & 0.85 & 1 \\
\hline
\end{tabular}

where $B$ is the face span of the excavation section.

345 (2) Monitoring data collection are arranged as shown in Fig. 5. Among them, point A, B, and C are the monitoring points for the settlement of the vault, DE and FH are the surrounding rock convergence line. The surrounding rock

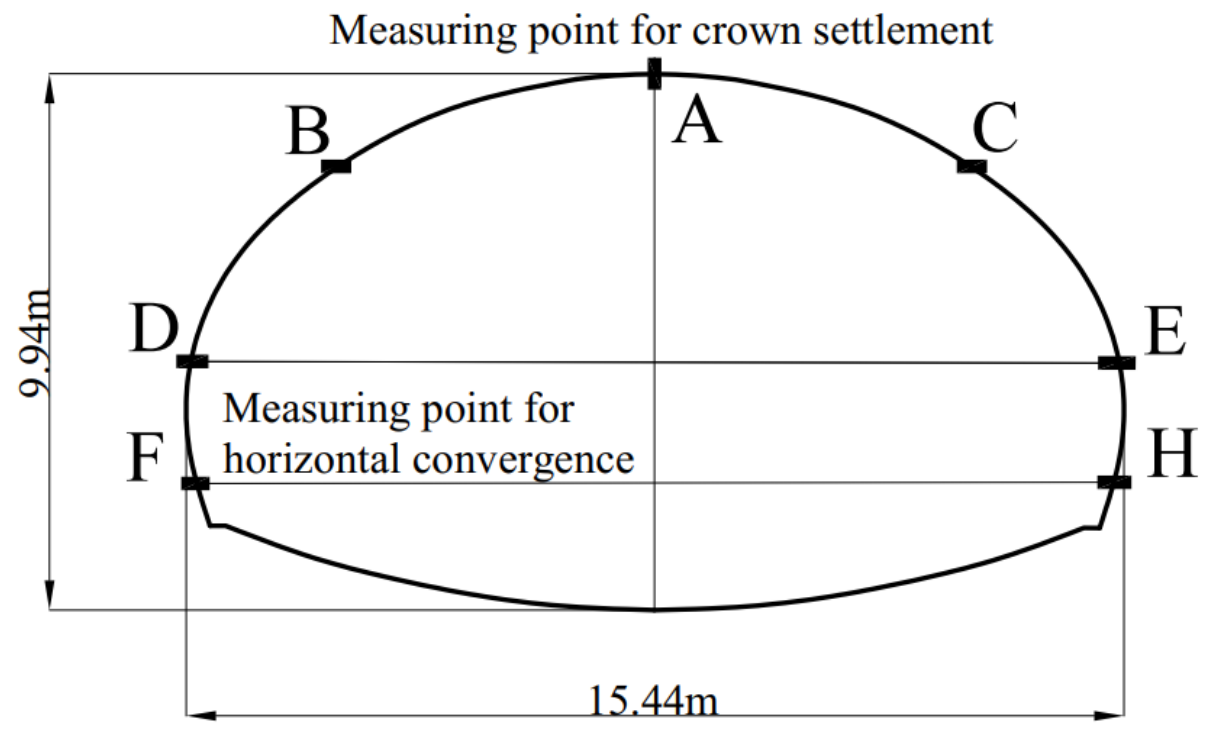

Fig. 5 Schematic diagram of monitoring point layout.

(3) Calculation of the tunneling collapse risk

According to Eq. (3), the cloud model parameter values $(E x, E n, H e)$ of the two monitoring indicators are constructed, as shown in Table 6. Finally, the tunnel collapse risk BPAs is constructed by Eq. (4).

The cloud model is used to obtain the BPAs of the cumulative settlement and daily settlement of the monitoring data (A, B, C, DE, and FH), and the improved D-S theory is used to fuse them 

collapse risk value, the flowchart as shown in Fig. 6 .

Table 6 Cloud models parameter value of the two monitoring indicators.

\begin{tabular}{ccccccccccccc}
\hline & I & \multicolumn{1}{c}{ II } & \multicolumn{1}{c}{ III } & \multicolumn{7}{c}{ IV } \\
\cline { 2 - 11 } Indicators & $E x$ & $E n$ & $H e$ & $E x$ & $E n$ & $H e$ & $E x$ & $E n$ & $H e$ & $E x$ & $E n$ & $H e$ \\
\hline $\begin{array}{c}\text { Daily } \\
\text { settlement }\end{array}$ & 1 & 0.333 & 0.002 & 3.5 & 0.5 & 0.002 & 7.5 & 0.833 & 0.002 & 12.5 & 0.833 & 0.002 \\
$\begin{array}{c}\text { Cumulative } \\
\text { settlement }\end{array}$ & 25 & 8.333 & 0.002 & 75 & 8.333 & 0.002 & 150 & 16.777 & 0.002 & 250 & 16.777 & 0.002 \\
\hline
\end{tabular}
risk of tunnel collapse.

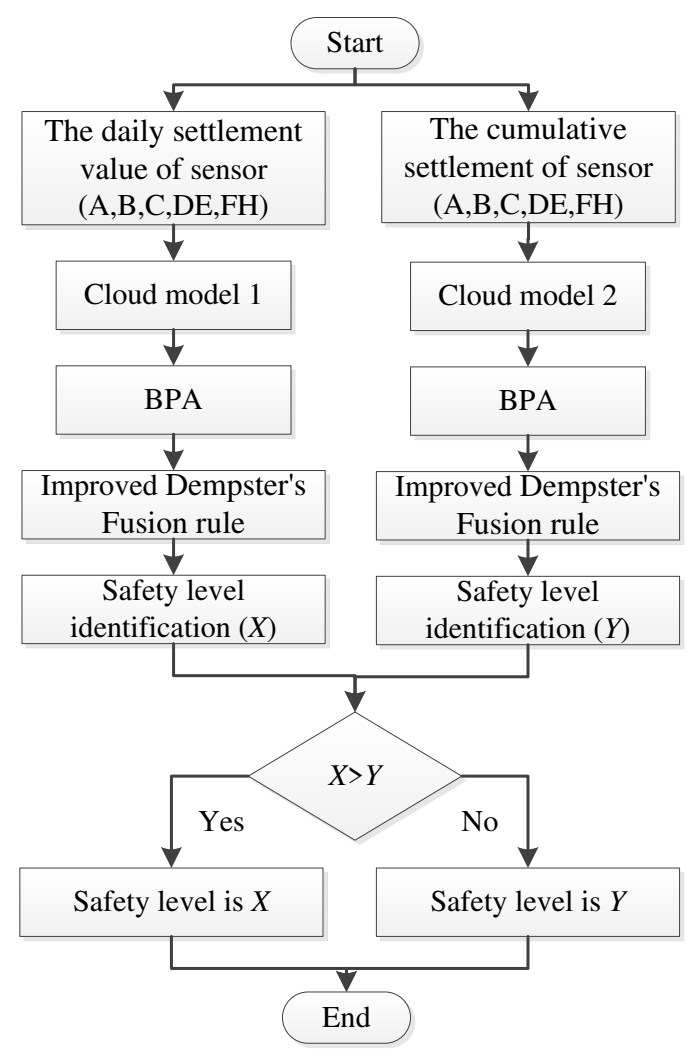

Fig. 6 Flowchart of monitoring data processing.

According to the flowchart shown in Fig. 6, The monitoring data from the above 20 sections were used for collapse risk assessment, the result as shown in Table 7. Obviously, in tunnel sections (No.6 and No.9), the probability of tunnel collapse for class I ( 0.50$)$ and class II ( 0.50$)$ is very close, which means that the results are very uncertain. It is difficult to make an accurate judgment on the 
Table 7 Results of Cloud model at ten monitoring sections.

\begin{tabular}{cccccccc}
\hline Tunnel section & $m(\mathrm{I})$ & $m(\mathrm{II})$ & $m(\mathrm{III})$ & $m(\mathrm{IV})$ & $m(\Theta)$ & Predicted level & Ture level \\
\hline No.1 & $\mathbf{0 . 5 2}$ & 0.46 & 0.00 & 0.00 & 0.02 & I & II \\
No.2 & 0.00 & 0.00 & $\mathbf{1 . 0 0}$ & 0.00 & 0.00 & III & III \\
No.3 & 0.48 & $\mathbf{0 . 5 0}$ & 0.00 & 0.00 & 0.02 & II & II \\
No.4 & 0.00 & $\mathbf{0 . 9 5}$ & 0.00 & 0.00 & 0.05 & II & II \\
No.5 & 0.01 & $\mathbf{0 . 9 7}$ & 0.00 & 0.00 & 0.02 & II & II \\
No.6 & $\mathbf{0 . 5 0}$ & $\mathbf{0 . 5 0}$ & 0.00 & 0.00 & 0.00 & -- & II \\
No.7 & 0.00 & $\mathbf{1 . 0 0}$ & 0.00 & 0.00 & 0.00 & II & II \\
No.8 & $\mathbf{1 . 0 0}$ & 0.00 & 0.00 & 0.00 & 0.00 & I & I \\
No.9 & $\mathbf{0 . 5 0}$ & $\mathbf{0 . 5 0}$ & 0.00 & 0.00 & 0.00 & -- & I \\
No.10 & 0.00 & $\mathbf{0 . 9 9}$ & 0.00 & 0.00 & 0.01 & II & I \\
No.11 & 0.41 & $\mathbf{0 . 5 3}$ & 0.00 & 0.00 & 0.06 & II & II \\
No.12 & 0.00 & $\mathbf{0 . 6 0}$ & 0.35 & 0.00 & 0.05 & II & III \\
No.13 & $\mathbf{0 . 9 6}$ & 0.00 & 0.00 & 0.00 & 0.04 & I & I \\
No.14 & $\mathbf{1 . 0 0}$ & 0.00 & 0.00 & 0.00 & 0.00 & I & I \\
No.15 & 0.03 & $\mathbf{0 . 9 0}$ & 0.00 & 0.00 & 0.07 & II & II \\
No.16 & 0.58 & $\mathbf{0 . 4 0}$ & 0.00 & 0.00 & 0.02 & II & I \\
No.17 & 0.00 & $\mathbf{0 . 9 3}$ & 0.00 & 0.04 & 0.03 & II & II \\
No.18 & $\mathbf{1 . 0 0}$ & 0.00 & 0.00 & 0.00 & 0.00 & I & I \\
No.19 & $\mathbf{0 . 9 8}$ & 0.00 & 0.00 & 0.00 & 0.02 & I & I \\
No.20 & 0.00 & $\mathbf{1 . 0 0}$ & 0.00 & 0.00 & 0.00 & II & II \\
\hline
\end{tabular}

\subsection{Multi-source information fusion}

378 and reduce uncertainty in the tunnel collapse risk evaluation. Only section evaluation error appears

379 at section No. 9, indicating that the evaluation accuracy rate of 20 sections has reached $95 \%$. The confidence indexes $m(\Theta)$ of the 20 tunnel sections are all close to 0 , which means that the uncertainty 
of the results is 0 .

(2) The proposed method can solve the problem of inconsistent results of the three risk assessment methods effectively. For example, because the results of the three risk categories are different (SVM and CM belong to level I and BN belong to level II), the single-source risk assessment method cannot directly assess the overall tunnel collapse risk level of the monitoring section 4. The multi-source information fusion method is used to evaluate the monitoring section 4 and the results are shown in Table 8. The BPAs value of tunnel collapse risk level II (that is $m$ (II)) is equal to 1 , which means that the collapse risk level for the monitoring section 4 is level II with a high confidence level

(3) Among those sections, the collapse risk values of sections No.2 and No.12 are III (Smallscale collapse), and the rest are level I (Safe) or level II (Deformation). This indicates that small collapses are likely to occur in sections No.2 and No.12 during the excavation stage. It is necessary to strengthen support levels and supervision to reduce the risk of collapse.

Table 8 Results of multi-source information fusion at ten monitoring sections.

\begin{tabular}{cccccccc}
\hline Tunnel section & $m(\mathrm{I})$ & $m(\mathrm{II})$ & $m(\mathrm{III})$ & $m(\mathrm{IV})$ & $m(\Theta)$ & Predicted level & Ture level \\
\hline No.1 & 0.00 & $\mathbf{0 . 9 9}$ & 0.01 & 0.00 & 0.00 & II & II \\
No.2 & 0.00 & 0.00 & $\mathbf{1 . 0 0}$ & 0.00 & 0.00 & III & III \\
No.3 & 0.00 & $\mathbf{1 . 0 0}$ & 0.00 & 0.00 & 0.00 & II & II \\
No.4 & 0.00 & $\mathbf{1 . 0 0}$ & 0.00 & 0.00 & 0.00 & II & II \\
No.5 & 0.00 & $\mathbf{1 . 0 0}$ & 0.00 & 0.00 & 0.00 & II & II \\
No.6 & 0.00 & $\mathbf{1 . 0 0}$ & 0.00 & 0.00 & 0.00 & II & II \\
No.7 & 0.00 & $\mathbf{1 . 0 0}$ & 0.00 & 0.00 & 0.00 & II & II \\
No.8 & $\mathbf{1 . 0 0}$ & 0.00 & 0.00 & 0.00 & 0.00 & I & I \\
No.9 & 0.17 & $\mathbf{0 . 8 3}$ & 0.00 & 0.00 & 0.00 & II & I \\
No.10 & $\mathbf{0 . 8 5}$ & 0.11 & 0.02 & 0.02 & 0.00 & I & I \\
No.11 & 0.00 & $\mathbf{1 . 0 0}$ & 0.00 & 0.00 & 0.00 & II & II \\
No.12 & 0.00 & 0.35 & $\mathbf{0 . 6 4}$ & 0.01 & 0.00 & III & III \\
No.13 & $\mathbf{1 . 0 0}$ & 0.00 & 0.00 & 0.00 & 0.00 & I & I \\
No.14 & $\mathbf{1 . 0 0}$ & 0.00 & 0.00 & 0.00 & 0.00 & I & I \\
No.15 & 0.00 & $\mathbf{1 . 0 0}$ & 0.00 & 0.00 & 0.00 & II & II \\
\hline
\end{tabular}




\begin{tabular}{llllllll}
\hline No.16 & $\mathbf{0 . 8 8}$ & 0.12 & 0.00 & 0.00 & 0.00 & I & I \\
No.17 & 0.00 & $\mathbf{1 . 0 0}$ & 0.00 & 0.00 & 0.00 & II & II \\
No.18 & $\mathbf{1 . 0 0}$ & 0.00 & 0.00 & 0.00 & 0.00 & I & I \\
No.19 & $\mathbf{1 . 0 0}$ & 0.00 & 0.00 & 0.00 & 0.00 & I & I \\
No. 20 & 0.00 & $\mathbf{1 . 0 0}$ & 0.00 & 0.00 & 0.00 & II & II \\
\hline
\end{tabular}

\subsection{Verification of evaluation results}

When the tunnel was excavated to section ZK243+143, the tunnel vault collapsed, as shown in

Fig. 7. This is due to the section being in the fracture zone of the surrounding rock and the

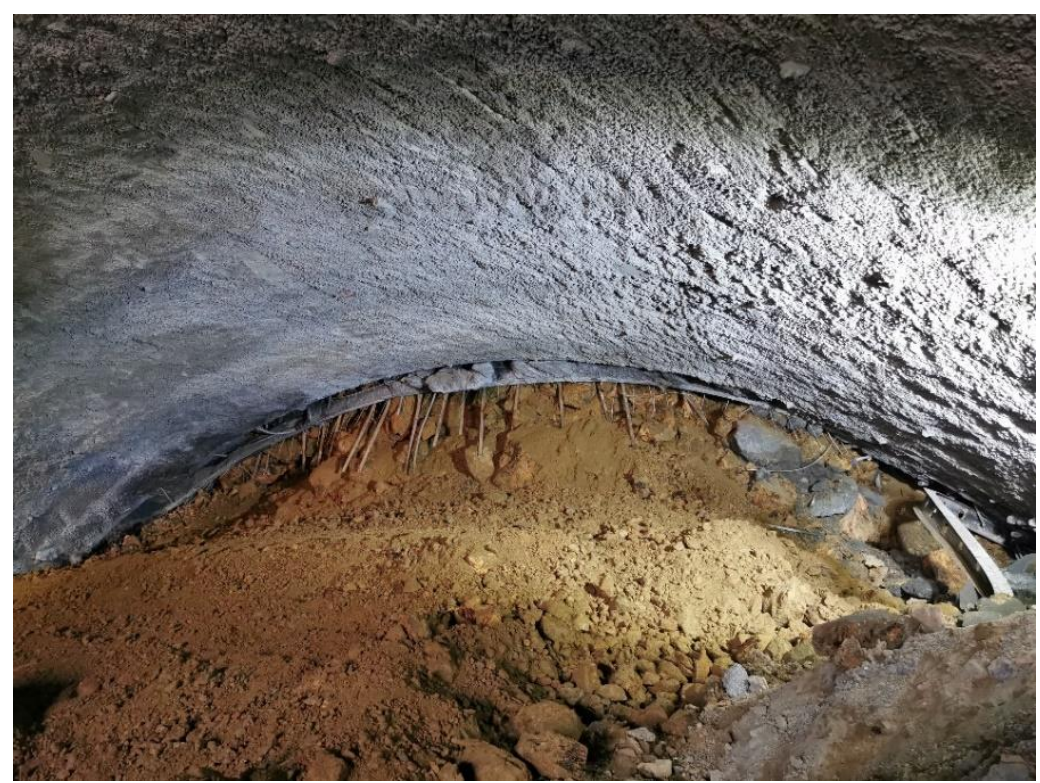

Fig. 7 Tunnel collapse 
In actual engineering, due to the influence of measurement errors and human factors, data from multi-source observations may have inevitable deviations. This article will use the MC simulation technology to simulate the uncertainty of the data. The factors affecting tunnel collapse are assumed to obey normal distribution. To further verify the robustness of the proposed hybrid method under unavoidable deviations, we added different deviation percentages (ie $5 \%, 10 \%, 15 \%$, and $20 \%$ ) to the collected data. In this paper, the number of repeated iterations $P$ is set to 1000 . Fig. 8 shows the results of tunnel collapse risk assessment after 1000 iterations for 4 tunnel sections (No. 1, 2, 3, and 8) at different offset levels. Fig. 9 shows the global sensitivity analysis about tunnel section No. 2. The following conclusions can be obtained:

(1) The proposed multi-source information fusion approach has good robustness to deviation. In order to better understand the bias, Fig. 8 shows the frequency of a certain collapse risk level after 1000 iterations under different biases in 4 tunnel sections (No.1, No.2, No.3, and No.8). When the percentage of bias is increased, the accuracy of the risk assessment will be slightly reduced, but it will remain at a high level. Obviously, all data with a deviation of less than $10 \%$ can almost achieve an evaluation accuracy rate close to $100 \%$, proving that the method is accurate and reliable under low bias. When the deviation is $20 \%$, the evaluation accuracy of all tunnel sections is still reached $100 \%$. This is because the results of the three single-information evaluation methods of tunnel section No. 3 are consistent (that is, the results of all three different models are risk level II higher than $90 \%$, which proves that the method has strong robustness under high deviations. Anymore, the accuracy of the assessment of the No. 3 tunnel section under each level of bias has (Deformation)), so no conflicting information will have a negative impact on the result of multi- 
source information fusion.

(2) Since the No. 2 tunnel section is in a dangerous state (Small-scale collapse), a global sensitivity analysis is performed on this part to find out the key risk factors that affect the tunnel collapse. Therefore, some measures to prevent tunnel collapse can be taken in advance. The Spearman's rank correlation coefficient (Eq. (12)) is used to measures the degree of influence of risk factors on the risk level of the tunneling collapse. As shown in Fig. 9, $X_{3}, X_{5}, X_{6}$, and $X_{11}$ are the top four risk factors that have the greatest impact on tunnel collapse. To reduce the risk level of tunnel section No.2, more attention should be paid to these four risk factors. In addition, when the deviation level increases to $20 \%$, the results of the most sensitive risk factors remain unchanged, again verifying the robustness of the proposed method.
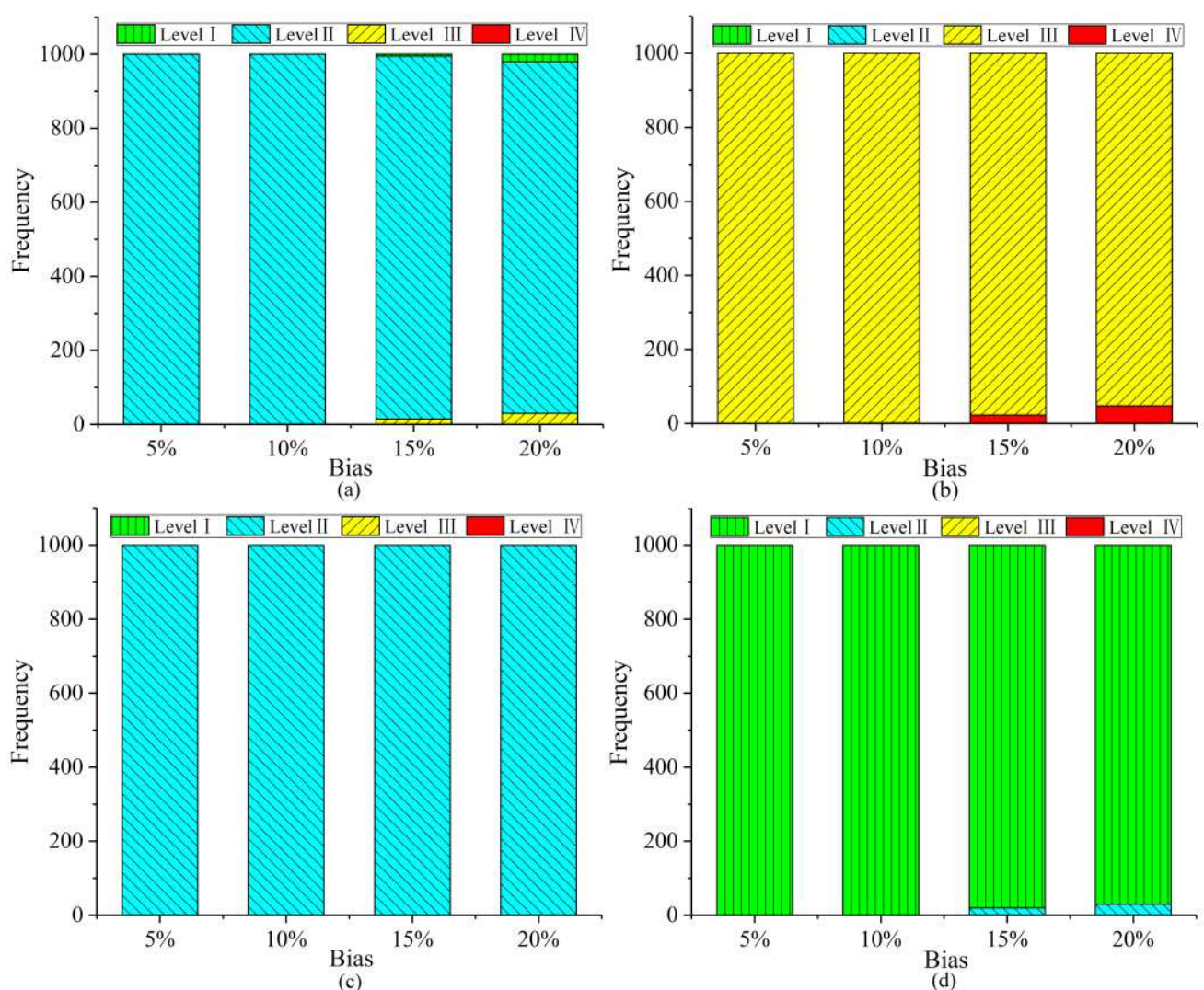
four section: (a) No.1; (b) No.2; (c) No.3; (d) No.8. 


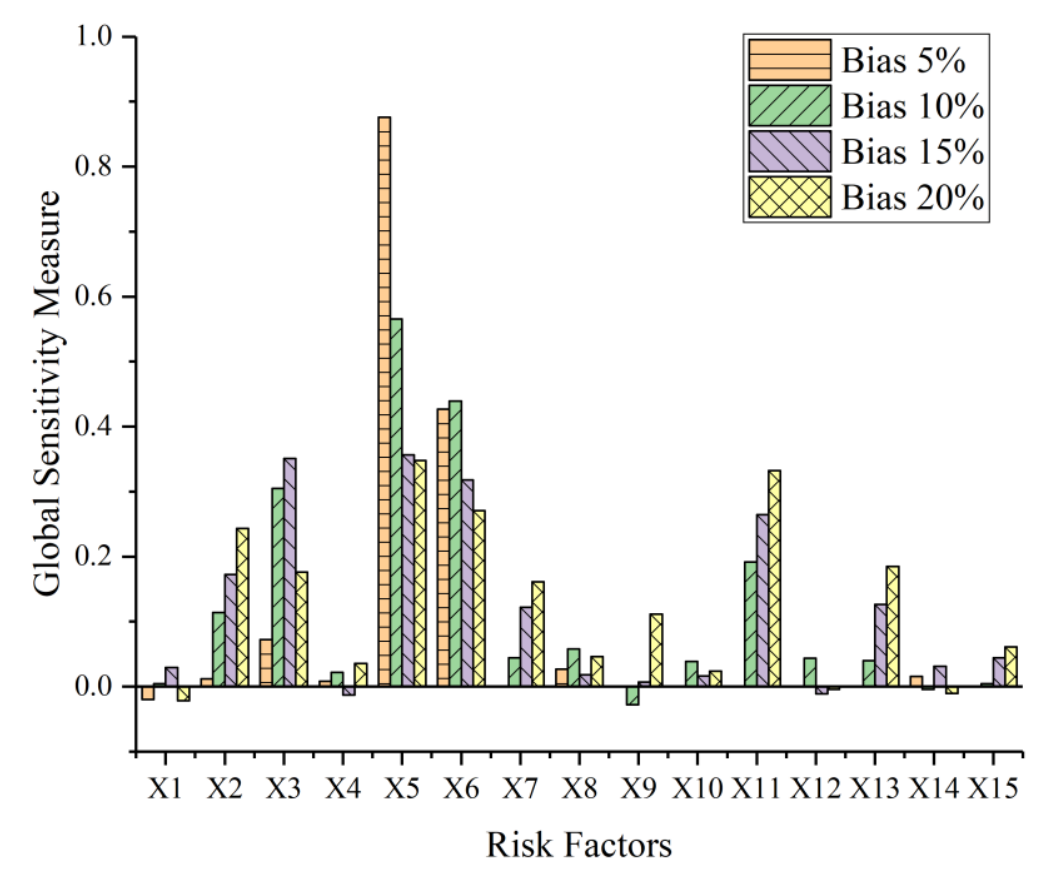

442

Fig. 9 Global sensitivity analysis of 15 risk indicators (No. 2 section)

\section{Discussion}

There is no doubt that the single-source information assessment method also can estimate the tunnel collapse risk level. However, the single source of information does not fully reflect the environment of the tunnel construction, resulting in a certain bias and low accuracy of the assessment results. To compare the single-source information evaluation method with the multisource information fusion method, the Monte Carlo simulation is used to simulate the inevitable uncertainty, and the four evaluation methods are calculated 1000 times. The tunneling collapse risk assessment results of four assessment methods iterate 1000 times under different deviation levels at tunnel section No.2, as shown in Fig. 10. The following conclusions can be obtained:

The single-source information assessment method (Fig. 10 (a), (b), and (c)) can get an accurate assessment result in case of small deviations, but it performs poorly at high bias. The multi-source 
information fusion method is more robust than the single-source information assessment method.

As seen in Fig. 10 (d), the multi-source information fusion method has a higher accuracy of assessment under a large bias, proving that the proposed method has good robustness. This is because the proposed method makes full use of available information, including contradictory information. When the data deviation is $20 \%$, the evaluation accuracy of the single-source information evaluation method is less than $80 \%$ in 1000 iterations. In order words, the single-source

461 information assessment method has a high sensibility to bias. However, the multi-source underground environment.
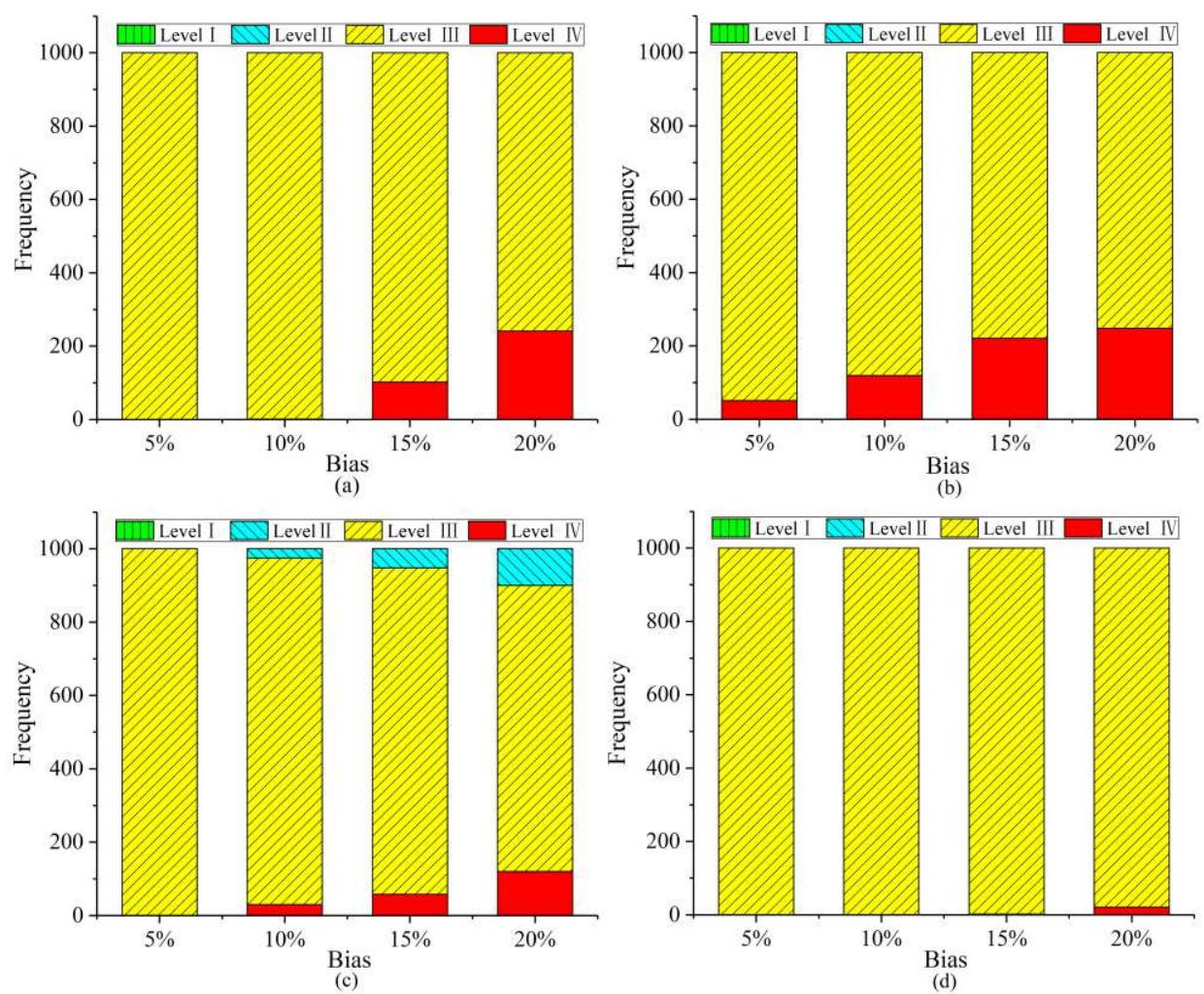
different deviation levels: (a) SVM; (b) BN; (c) CM; (d) Multi-source information fusion method. 


\section{Conclusions and future works}

This paper proposes a multi-source information fusion method for the tunneling collapse risk assessment, which provides risk warning and decision-making suggestions for tunnel excavation. The analysis process consists of four main steps: (1) Risk assessment systems are established for the three information sources (i.e. statistical data, physical sensors, and expert judgment provided by humans) separately; (2) The three information sources are processed by the BN, CM, and SVM respectively to obtain the BPAs of the collapse risk; (3) All predictions from three different assessment method are fused to obtain the overall tunneling collapse risk; (4) The Monte Carlo simulation method is used for global sensitivity analysis and robustness verification. Finally, the Jinzhupa tunnel in China is used to verify the applicability of the proposed approach. The methods developed in this research have the following innovations and capabilities:

(1) It can synthesize multi-source information to obtain a more accurate result for the tunneling collapse risk assessment. Due to many risk factors, the tunneling collapse risk assessment is a multiattribute decision-making problem. In this paper, both soft data from domain experts and hard data from electrical sensors and statistical data are used for evaluating the tunnel collapse risk. A hybrid combination rule combining the weighted mean rule and Dempster's rule is proposed to process multiple conflicting pieces of evidence. Besides, a confidence index, $m(\Theta)$ is adopted to measure the reliability of the tunnel collapse risk result. As shown in Table 8, the value of $m(\Theta)$ is zero, indicating that the tunneling collapse risk has a high degree of confidence.

(2) As the deviation level of input data increases, the accuracy rate of the single-source information evaluation method is gradually decreasing. However, the proposed multi-source 
information fusion method is very robust to deviations. Even when the deviation is $20 \%$, the accuracy of the collapse risk assessment still reaches $97.9 \%$. In other words, this method has excellent tolerance to bias, which eliminates the adverse effects of deviation to the maximum extent and ensures the accuracy and reliability of the evaluation results.

(3) When the tunnel section is in a dangerous state, in order to provide advice to decisionmakers, global sensitivity analysis is proposed to identify the most influential risk factors. The global sensitivity analysis considers the interaction between risk factors, making the results more in line with actual construction conditions. In the tunnel case of this study, the factors $X_{3}$ (Rock mass grade), $X_{5}$ (Unfavorable geology), $X_{6}$ (Bias angle), and $X_{11}$ (Timeliness of primary support) are identified to have the greatest impact on the risk of tunnel collapse. Besides, because measurement errors or human errors may cause data deviations, the MC method is used to simulate the data deviations to prove that the proposed method still has good robustness under deviations.

The proposed method in this paper also has some limitations. Experts are still required to participate in the entire evaluation process, which means that a truly automated evaluation has not yet been achieved. In terms of tunnel collapse data collection, the amount of data is still small, and a system needs to be developed to collect data on a global scale. In addition, this method cannot predict the risk status of the next construction process, and further research is needed.

\section{Data Availability Statement}

The datasets generated during and/or analyzed during the current study are not publicly available but are available from the corresponding author on reasonable request. 
This work was supported by the National Natural Science Foundation of China (Grant Nos.:

$51151678164 ;$ 51478118); Guangxi Natural Science Foundation (Grant Nos.: 2018GXNSFDA138009).

512 The authors would like to express the appreciation and thanks to the managers and San-Ming

513 Pu-Yan Expressway Co. LTD.

\section{Conflicts of Interest}

515 The authors declare no conflicts of interest.

516

517 


\section{References}

Balazs, J.A., Velásquez, J.D., 2016. Opinion Mining and Information Fusion: A survey. Information Fusion 27, 95-110. https://doi.org/10.1016/j.inffus.2015.06.002

Ding, L.Y., Zhou, C., 2013. Development of web-based system for safety risk early warning in urban metro construction. Automation in Construction 34, 45-55. https://doi.org/10.1016/j.autcon.2012.11.001

Guo, K., Zhang, L., 2021. Multi-source information fusion for safety risk assessment in underground tunnels. Knowledge-Based Systems 227 , 107210. https://doi.org/10.1016/j.knosys.2021.107210

Haroonabadi, H., Haghifam, M.-R., 2011. Generation reliability assessment in power markets using Monte Carlo simulation and soft computing. Applied Soft Computing 11, 5292-5298. https://doi.org/10.1016/j.asoc.2011.05.031

Hasanpour, R., Rostami, J., Schmitt, J., Ozcelik, Y., Sohrabian, B., 2020. Prediction of TBM jamming risk in squeezing grounds using Bayesian and artificial neural networks. Journal of Rock Mechanics and Geotechnical Engineering 12, 21-31. https://doi.org/10.1016/j.jrmge.2019.04.006

Huang, Y., 2009. Advances in Artificial Neural Networks - Methodological Development and Application. Algorithms 2, 973-1007. https://doi.org/10.3390/algor2030973

Janssen, H., 2013. Monte-Carlo based uncertainty analysis: Sampling efficiency and sampling convergence. Reliability Engineering \& System Safety 109, 123-132. https://doi.org/10.1016/j.ress.2012.08.003 
Kou, G., Lu, Y., Peng, Y., Shi, Y., 2012. Evaluation of classification algorithms using mcdm and rank correlation. Int. J. Info. Tech. Dec. Mak. 11, 197-225. https://doi.org/10.1142/S0219622012500095

Leung, Y., Ji, N.-N., Ma, J.-H., 2013. An integrated information fusion approach based on the theory of evidence and group decision-making. Information Fusion 14, 410-422. https://doi.org/10.1016/j.inffus.2012.08.002

Li, D., Liu, C., Gan, W., 2009. A new cognitive model: Cloud model. Int. J. Intell. Syst. 24, 357375. https://doi.org/10.1002/int.20340

Li, N., Feng, X., Jimenez, R., 2017. Predicting rock burst hazard with incomplete data using Bayesian networks. Tunnelling and Underground Space Technology 61, 61-70. https://doi.org/10.1016/j.tust.2016.09.010

Li, S., Liu, C., Zhou, Z., Li, L., Shi, S., Yuan, Y., 2021. Multi-sources information fusion analysis of water inrush disaster in tunnels based on improved theory of evidence. Tunnelling and Underground Space Technology 113, 103948. https://doi.org/10.1016/j.tust.2021.103948

Liu, K., Liu, B., 2019. Intelligent information-based construction in tunnel engineering based on the GA and CCGPR coupled algorithm. Tunnelling and Underground Space Technology 88, 113128. https://doi.org/10.1016/j.tust.2019.02.012

Liu, Y., Lian, J., Bartolacci, M.R., Zeng, Q.-A., 2014. Density-Based Penalty Parameter Optimization on C-SVM. The Scientific World Journal 2014, 1-9. https://doi.org/10.1155/2014/851814

Pan, Y., Zhang, L., 2021. Roles of artificial intelligence in construction engineering and management: A critical review and future trends. Automation in Construction 122, 103517. 
Pan, Y., Zhang, L., Wu, X., Skibniewski, M.J., 2020. Multi-classifier information fusion in risk analysis. Information Fusion 60, 121-136. https://doi.org/10.1016/j.inffus.2020.02.003

Qian, Y., Liang, J., Yao, Y., Dang, C., 2010. MGRS: A multi-granulation rough set. Information Sciences, Special Issue on Modelling Uncertainty 180, 949-970. https://doi.org/10.1016/j.ins.2009.11.023

Qiao, S., Cai, Z., Tan, J., Xu, P., Zhang, Y., 2020. Analysis of Collapse Mechanism and Treatment

Robert, C.P., Casella, G., 2004. Monte Carlo Statistical Methods, Springer Texts in Statistics. Springer New York, New York, NY. https://doi.org/10.1007/978-1-4757-4145-2

Satpal, S.B., Guha, A., Banerjee, S., 2016. Damage identification in aluminum beams using support vector machine: Numerical and experimental studies: Damage Identification in Al Beams https://doi.org/10.1002/stc.1773

Wang, B., Li, S., Zhang, Qianqing, Li, L., Zhang, Qian, Xu, F., 2016. Risk Assessment of a Tunnel Collapse in a Mountain Tunnel Based on the Attribute Synthetic Evaluation System, in: GeoChina 2016. Presented at the Fourth Geo-China International Conference, American Society of Civil Engineers, Shandong, China, pp. 198-209. https://doi.org/10.1061/9780784480038.025

Wang, S., Li, L., Shi, S., Cheng, S., Hu, H., Wen, T., 2020. Dynamic Risk Assessment Method of Collapse in Mountain Tunnels and Application. Geotech Geol Eng 38, 2913-2926. https://doi.org/10.1007/s10706-020-01196-7 
583

584

585

586

587

588

589

590

591

592

593

594

595

596

597

598

599

600

601

602

603

604

Worden, K., Manson, G., 2007. The application of machine learning to structural health monitoring. Phil. Trans. R. Soc. A. 365, 515-537. https://doi.org/10.1098/rsta.2006.1938

Wu, X., Liu, H., Zhang, L., Skibniewski, M.J., Deng, Q., Teng, J., 2015. A dynamic Bayesian network based approach to safety decision support in tunnel construction. Reliability Engineering \& System Safety 134, 157-168. https://doi.org/10.1016/j.ress.2014.10.021

Yager, R.R., 2016. Multi-source Information Fusion Using Measure Representations, in: SamingerPlatz, S., Mesiar, R. (Eds.), On Logical, Algebraic, and Probabilistic Aspects of Fuzzy Set Theory, Studies in Fuzziness and Soft Computing. Springer International Publishing, Cham, pp. 199-214. https://doi.org/10.1007/978-3-319-28808-6_12

Zhang, G.-H., Chen, W., Jiao, Y.-Y., Wang, H., Wang, C.-T., 2020. A failure probability evaluation method for collapse of drill-and-blast tunnels based on multistate fuzzy Bayesian network. Engineering Geology 276, 105752. https://doi.org/10.1016/j.enggeo.2020.105752

Zhang L, Wu X, Miroslaw J, et al. Bayesian-Network-Based Safety Risk Analysis in Construction Projects[J]. Reliability Engineering \& System Safety, 2014, 131: 29-39. https://doi.org/10.1016/j.ress.2014.06.006

Zhang, L., Wu, X., Zhu, H., AbouRizk, S.M., 2017. Perceiving safety risk of buildings adjacent to tunneling excavation: An information fusion approach. Automation in Construction 73, 88101. https://doi.org/10.1016/j.autcon.2016.09.003

Zhang, P., Li, T., Wang, G., Luo, C., Chen, H., Zhang, J., Wang, D., Yu, Z., 2021. Multi-source information fusion based on rough set theory: A review. Information Fusion 68, 85-117. https://doi.org/10.1016/j.inffus.2020.11.004

Zhou, C., Yin, K., Cao, Y., Ahmed, B., 2016. Application of time series analysis and PSO-SVM 
605

606

607

608

609

610

611

612

613

614

model in predicting the Bazimen landslide in the Three Gorges Reservoir, China. Engineering Geology 204, 108-120. https://doi.org/10.1016/j.enggeo.2016.02.009

Zhou, F., 2008. Research on Fuzzy Hierarchical Evaluation of Mountain Tunnel Landslide Risk. Master's Thesis, Central South University, China, Changsha

Sun, J., 2019. Study on collapse risk and stability evaluation in mining construction of mountain tunnel. Master's Thesis, Beijing Jiaotong University, China, Beijing

JTG/T 3660-2020. Technical specification for construction of highway tunnel

DB 35/T 1067-2010. Technical code for monitoring measurement of highway tunnel 